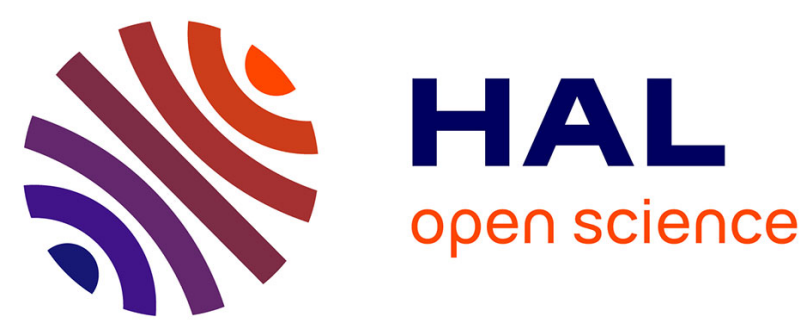

\title{
TRANSMISSION ACOUSTIQUE PAR DES STRUCTURES SANDWICHS DE TYPE ARIANE V : PRISE EN COMPTE DES EFFETS D'ÉCRASEMENT ET DE CISAILLEMENT TRANSVERSE
}

\author{
A. Blaise, C. Lesueur
}

\section{To cite this version:}

A. Blaise, C. Lesueur. TRANSMISSION ACOUSTIQUE PAR DES STRUCTURES SANDWICHS DE TYPE ARIANE V : PRISE EN COMPTE DES EFFETS D'ÉCRASEMENT ET DE CISAILLEMENT TRANSVERSE. Journal de Physique IV Proceedings, 1992, 02 (C1), pp.C1-499-C1-502. 10.1051/jp4:19921107 . jpa-00251061

\section{HAL Id: jpa-00251061 https://hal.science/jpa-00251061}

Submitted on 1 Jan 1992

HAL is a multi-disciplinary open access archive for the deposit and dissemination of scientific research documents, whether they are published or not. The documents may come from teaching and research institutions in France or abroad, or from public or private research centers.
L'archive ouverte pluridisciplinaire HAL, est destinée au dépôt et à la diffusion de documents scientifiques de niveau recherche, publiés ou non, émanant des établissements d'enseignement et de recherche français ou étrangers, des laboratoires publics ou privés. 
TRANSMISSION ACOUSTIQUE PAR DES STRUCTURES SANDWICHS DE TYPE ARIANE V : PRISE EN COMPTE DES EFFETS D'ÉCRASEMENT ET DE CISAILLEMENT TRANSVERSE

\author{
A. BLAISE et C. LESUEUR \\ Laboratoire Vibrations - Acoustique, INSA Lyon Bat. 303, 20 avenue A. Einstein, \\ F-69621 Villeurbanne cedex, France
}

\begin{abstract}
The main goal of vibroacoutic studies on the Ariane $V$ launch vehicle, multilayered orthotropic cylindrical structure is to achieve low magnitude internal acoustic environment. This can be do, $|1|-|2|$, without increasing the mass per unit area, by increasing the core thickness of the three layered basic structure. But this reduce the transverse structure stiffness; so it is of first interest and importance to examine if the classical two dimensionnal (2D) hypothesis is a good approximation; or if the differencies on the transmission loss (T.L) between the cases 2D and 3D (three-dimensionnal theorie including transverse thickness deformation) are very noticeable. Thus, we develop a new vibroacoustical analytical model including for each layer a 3D field displacement; and we present some results for the T.L 2D - 3D comparison for a three layered Ariane V configuration.
\end{abstract}

\title{
$1 /$ Position du_problème.
}

Les structures sensibles de la partie haute des lanceurs sont de type cylindrique multicouche et sont excitées par un champ aérien intense. Nous avons montré lors de précédentes études $|1|-|2|$ que leur optimisation acoustique était possible, à masse surfacique constante, par augmentation de l'épaisseur de l'âme. La diminution de la raideur transverse est une conséquence dont les effets ne sont pas pris en compte dans les théories classiques bidimensionnelles (2D) que ce soit pour les modèles de contraintes planes $13 \mid$ ou pour des modèles incluant le cisaillement transverse $|2|$ sur le plan vibro-acoustique et $|4|$ d'un point de vue purement vibratoire. Il nous semble par conséquent intéressant d'évaluer le degré d'approximation de telles théories (2D) en comparant leurs résultats : indices d'affaiblissement acoustique à ceux issus d'une théorie tridimensionnelle (3D) incluant les effets d'écrasement transverse. En d'autres termes, les théories 2D sont-elles suffisantes ou au contraire faut-il pour de telles structures utiliser une théorie 3D ? Pour répondre à cette question, nous adoptons une modélisation de la structure multicouche, figure 1, orthotrope infinie incluant les effets de membrane, flexion, cisaillement et écrasement transverse de chaque couche. Le tout étant excité par une onde plane harmonique d'incidence $\left(\theta_{\mathrm{I}}, \Psi_{\mathrm{I}}\right)$ dans le milieu extérieur I, le milieu intérieur (II) est de type non résonant. La méthode de résolution employée est de type propagative en milieux semi-infinis.

\section{2/ Formulation théorique "30".}

Elle se fait en deux temps; le premier concerne la mise en équation du problème vibratoire d'une couche i sollicitée sur ses faces interne et externe, le second concerne la résolution du problème vibroacoustique de la structure multicouche couplée aux milieux fluides.

2.1. Problème vibratoire : La couche $i$ est sollicitée en ces faces externes $R_{i}^{+}$et interne $R_{i}^{-}$par soit des forces par unité de surface $\left\{\mathrm{iF}_{\mathrm{e}}^{+}\right\} ;\left\{\mathrm{iF}_{\mathrm{e}}^{-}\right\}$lorsque l'interface est de type structure-structure ou $\mathrm{P}_{\mathrm{I}} \overrightarrow{\mathrm{n}^{+}} ; \mathrm{P}_{\mathrm{II}^{-}} \overrightarrow{\mathrm{n}}^{-}$si l'interface est de type fluide-structure. Le champ de déplacement axial, circonférentiel et radial d'un point $\mathrm{M}_{i}$ de la couche est définie en fonction du champ de déplacements d'un point $\mathrm{N}_{i}$ de la surface moyenne et de quatre fonctions : ${ }^{i} \varphi_{1}=1,2$ traduisant le cisaillement transverse et $i \psi_{1} 1=1,2$ traduisant l'effet d'écrasement transverse linéaire $(1=1)$ et parabolique $(l=2)$, avec $\left(r_{i}, \theta_{i}, x_{i}\right)$ les coordonnées cylindriques locales, par : 


$$
\begin{aligned}
& \mathrm{i} U_{1}\left(M_{i}, t\right)=i U_{1}\left(N_{i}, t\right)+r_{i}\left(-\frac{\partial^{i} U_{3}\left(N_{i}, t\right)}{\partial z_{i}}+i \varphi_{1}\left(N_{i}, t\right)\right) \\
& i U_{2}\left(M_{i}, t\right)=i U_{2}\left(N_{i}, t\right)+\frac{r_{i}}{R_{i}}\left\{-\frac{\partial^{i} U_{3}\left(N_{i}, t\right)}{\partial \theta_{i}}+i U_{2}\left(N_{i}, t\right)+i \varphi_{2}\left(N_{i}, t\right)\right\} \\
& i U_{3}\left(M_{i}, t\right)=i U_{3}\left(N_{i}, t\right)+r_{i} \cdot i \Psi_{1}\left(N_{i}, t\right)+\frac{r_{i}^{2}}{2} i \Psi_{2}\left(N_{i}, t\right)
\end{aligned}
$$

Le champ des petites déformations est calculé d'après les relations classiques $i \varepsilon_{k 1}=\frac{1}{2}\left(i U_{k, 1}+i U_{l, k}\right)$ exprimées en coordonnées cylindriques ; celui des contraintes est obtenu en considérant un matériau orthotrope viscoélastique tel que les axes d'orthotropie sont confondus avec ceux du cylindre, soit $i \overline{\bar{\sigma}}=i \mathrm{C} \overline{\bar{\varepsilon}}$ tel que $\mathrm{iC}_{\mathrm{kl}}=\mathrm{i} \overline{\mathrm{C}}_{\mathrm{kl}}\left(1+\mathrm{j}\right.$ i $\left.\eta_{\mathrm{kl}}\right)$ avec $\mathrm{i} \overline{\mathrm{C}}_{\mathrm{kl}}$ : partie réelle de $\mathrm{i} \mathrm{C}_{\mathrm{kl}}, \mathrm{i}_{\mathrm{kl}}:$ facteur de perte. Ces éléments nous permettent de définir une fonctionnelle d'énergie $(F)$ qui s'exprime après integration sur la variable d'épaisseur à partir de sept fonctions indépendantes. L'extrémalisation de $F$ nous donne le système d'équations d'équilibre de la couche i couplée à son environnement. Ce qui, dans le cas d'une excitation par onde plane harmonique et après avoir développé le champ de déplacements sur la base des modes circonférentiels n, nous donne :

$$
\sum_{n=0}^{\infty}\left[\mathrm{iL}_{\mathrm{pq}}\right]\left\{\mathrm{iD}_{\mathrm{q}}^{\mathrm{n}}\right\} \cdot \exp \left[-\mathrm{j}^{\mathrm{i}} \mathrm{k}_{\mathrm{z}} \cdot \mathrm{z}_{\mathrm{i}}\right]=\left\{\mathrm{i} \mathrm{F}_{\mathrm{p}}^{\mathrm{ext}}\left(\mathrm{N}_{\mathrm{i}}\right)\right\}
$$

avec $\left[{ }^{i} L_{p q}\right]$ opérateur incluant les effets de masse ,de raideur et d'amortissement de la couche $i$ orthotrope, $\left\{\mathrm{i}_{\mathrm{q}}^{\mathrm{n}}\right\}^{\mathrm{t}}=\left[\mathrm{i} \mathrm{U}_{1}^{\mathrm{n}} \mathrm{j} \cos \mathrm{n} \theta_{\mathrm{i}}, \ldots, \mathrm{i} \mathrm{U}_{2}^{\mathrm{n}} \sin n \theta_{\mathrm{i}}, \ldots, \mathrm{i} \mathrm{U}_{3}^{\mathrm{n}} \cos \mathrm{n} \theta_{\mathrm{j}}\right],{ }_{\mathrm{i}} \mathrm{F}_{\mathrm{p}}^{\text {ext }}$ amplitude complexe de la résultante des sollicitations externes ramenée en $\mathrm{N}_{\mathrm{i}}$ et $\mathbf{i k}_{\mathbf{z}}$ le nombre d'onde axial.

2.2. Problème vibroacoustique : L'assemblage des différentes couches $\mathrm{i}=1$ à $\mathrm{N}$ et des deux milieux fluides est réalisé en considérant les continuités des champs de contraintes (normales et tangentielles) et de déplacements aux différents interfaces. Dans le cas d'une excitation par onde plane d'amplitude $\mathrm{P}_{0}$, la structure génère une onde réfléchie d'amplitude modale $A_{n}$ et transmise au milieu II $B_{n}$, considéré comme non résonnant . Ces amplitudes sont solutions du système linéaire modal défini $\operatorname{par}[\mathrm{A}]\{\mathrm{y}\}=\{\mathrm{F}\}$ de $\operatorname{rang}$ $(10 \mathrm{~N}-1)$ avec $\{y\}^{\mathrm{t}}=\left[\mathrm{A}_{\mathrm{n}},\left\{\mathrm{ND}_{\mathrm{q}}\right\},\{\mathrm{N}-1 \sigma\}, \ldots \ldots \ldots,\{1 \sigma\},\left\{1 \mathrm{D}_{\mathrm{q}}\right\}, \mathrm{B}_{\mathrm{n}}\right]$. Après résolution numérique de ce système, nous calculons l'indice d'affaiblissement acoustique TL ( qui diffère du résultat classique connu

pour les structures minces $12 \mathrm{l})$ défini par : $\operatorname{TL}\left(\omega, \theta_{\mathrm{I}}, \Psi_{\mathrm{I}}\right)=-10 \log _{10} \sum_{n=0}^{\infty} \tau_{n}\left(\omega, \theta_{I}, \psi_{I}\right)$

$$
\tau_{n}\left(\omega, \theta_{I}, \psi_{I}\right)=\frac{2 \rho_{I} \cdot C_{I}}{\varepsilon_{n} \cdot \rho_{I I} \cdot \omega \cdot R_{N}^{+} \cdot \cos \theta_{1}} \cdot \frac{\left|B_{n}\right|^{2}}{P_{0}^{2}}
$$

avec $\varepsilon_{\mathrm{n}}$ facteur de Neumann ; $\mathrm{P}_{\mathrm{I}}, \rho_{\mathrm{II}}$ masse volumique des milieux fluides. $\mathrm{C}_{\mathrm{I}}$ : célérité du son dans le milieu I.

\section{Résultats numériques.}

Nous traitons le cas d'une structure tricouche de type Ariane V en fibre de carbone-nid d'abeille-fibre de carbone dont les caractéristiques sont précisées dans le tableau 1, pour une incidence $\theta_{I}=45^{\circ} \psi_{I}=0$; nous comparons les résultats issus de notre théorie $2 \mathrm{D}|2|$ pour :

a) un modèle en contraintes planes sans cisaillement transverse, (2Dcp)

b) un modèle général avec cisaillement transverse (2D),

et ceux de la présente théorie 3D avec écrasement transverse (3D). 
Nous comparons figure 2, les résultats issus des théories $2 \mathrm{D}$ uniquement en ayant fait varier les coefficients d'élasticité de l'âme relatifs au cisaillement transverse ${ }^{2} C_{55}$ et ${ }^{2} C_{66}$. Les résultats obtenus montrent une différence minime pour $\mathrm{f}<\mathrm{f}_{\mathrm{a}}$ (fréquence d'anneau de la structure) de l'ordre de 1 à $2 \mathrm{~dB}$ hors zone de zéro d'impédance, une différence notable pour $f>f_{a}$ de l'ordre de $5 \mathrm{~dB}$ et pour $\mathrm{f}>\mathrm{f}_{\text {coin }}^{1}$ (fréquence de coincidence théorique $2 \mathrm{D}$ contraintes planes) de l'ordre de $10 \mathrm{~dB}$ à $40 \mathrm{~dB}$ à $2000 \mathrm{~Hz}$. Nous comparons, figure 3, les résultats relatifs aux théories 2D et 3D en ayant fixé la valeur du coefficient d'élasticité transverse de l'âme ${ }^{2} \mathrm{C}_{33}$. Nous faisons un constat analogue au précédent, avec pour

$\mathrm{f}_{\mathrm{a}}<\mathrm{f}<\mathrm{f}_{\text {coin }}^{1}: \mathrm{TL}_{3 \mathrm{D}}<\mathrm{TL} 2 \mathrm{D}$ et pour $\mathrm{f}>\mathrm{f}_{\text {coin }}^{1} \mathrm{TL}_{2 \mathrm{DCP}}<\mathrm{TL}_{3 \mathrm{D}}<\mathrm{TL}_{2 \mathrm{D}}$, linfluence de ${ }^{2} \mathrm{C}_{55}$ et $2 \mathrm{C}_{66}$ sur la variation de TL est moindre pour la théorie 3D que 2D.

Enfin, figure 3, nous analysons l'influence de ${ }^{2} \mathrm{C}_{33}$ de l'âme sur les écarts de TL, toutes choses égales par ailleurs. On relève des différences au niveau du TL et de la position de la singularité fréquentielle $\mathrm{f}_{\text {coin }}$ qui sont fonctions de la théorie employée : $2 \mathrm{D}$ ou $3 \mathrm{D}$.

\section{Conclusions.}

Pour la configuration tricouche avec âme en nid d'abeille étudiée et non optimisée, les résultats obtenus mettent en évidence l'influence relative des termes $2 C_{55}{ }^{2} C_{66}{ }^{2} C_{33}$ sur le niveau de $T L$ et sur la position de la singularité fréquentielle $f_{\text {coin. }}$. Par ailleurs, les résutlats issus d'une théorie $2 \mathrm{D}$ avec contraintes planes sont pour $f \leq f_{\text {coin }}$ toujours otpimistes vis à vis des modèles $2 D$ ou $3 D$. La réponse à notre question est sans ambiguité dans le domaine des basses fréquences, $f \leq f_{a}$ où les écarts sont limités à $2 \mathrm{~dB}$; pour le domaine fréquentiel $f>f_{a}$, il nous faut impérativement caractériser expérimentalement les $2 C_{i j}$ précités avant d'apporter une réponse spécifique à une géométrie donnée.

Tableau 1 : Configuration tricouches symétriques de rayon $\bar{R}=2 \mathrm{~m}$ avec $\rho_{1}=\rho_{3}=1483 \mathrm{~kg} / \mathrm{m}^{3}$,

$$
h_{1}=h_{3}=0.910^{-3} \mathrm{~m}, \rho_{2}=90.5 \mathrm{~kg} / \mathrm{m}^{3}, \mathrm{~h}_{2}=24.10^{-3} \mathrm{~m}
$$

\begin{tabular}{|c|c|c|c|c|c|c|c|c|c|c|}
\hline \multirow[t]{2}{*}{ Théorie } & \multirow{2}{*}{$\begin{array}{l}\text { Couche } \\
\text { (i) }\end{array}$} & \multicolumn{9}{|c|}{ Coefficients ${ }^{i C_{\rho \rho}} \times 10^{-10} \mathrm{~Pa}$} \\
\hline & & $\mathrm{C}_{11}$ & $\mathrm{C}_{12}$ & $\mathrm{C}_{13}$ & $\mathrm{C}_{22}$ & $\mathrm{C}_{23}$ & $\mathrm{C}_{33}$ & $\mathrm{C}_{44}$ & $\mathrm{C}_{55}$ & $\mathrm{C}_{66}$ \\
\hline \multirow[t]{2}{*}{$2 \mathrm{Dcp}$} & $1-3$ & 6.83 & 0.683 & & 2.28 & 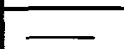 & 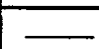 & 1.17 & - & - \\
\hline & 2 & $10^{-4}$ & $0.310^{-4}$ & & $10^{-4}$ & $\underline{-}$ & 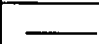 & $0.410^{-4}$ & - & - \\
\hline \multirow{2}{*}{$2 \mathrm{D}$} & $1-3$ & 7.73 & 1.04 & & 2.14 & 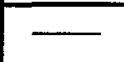 & 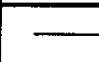 & 1.24 & 2.5 & 2.5 \\
\hline & 2 & $10^{-4}$ & $0.310^{-4}$ & & $10^{-4}$ & & & $0.410^{-4}$ & 0.023 & 0.037 \\
\hline \multirow{2}{*}{ D } & $1-3$ & 7.73 & 1.04 & 2.63 & 2.41 & 1.04 & $\overline{7.73}$ & 1.24 & 2.5 & 2.5 \\
\hline & 2 & $10^{-4}$ & $0.310^{-4}$ & $0.310^{-4}$ & $10^{-4}$ & $0.310^{-4}$ & 0.138 & $0.410^{-4}$ & 0.023 & 0.037 \\
\hline
\end{tabular}

\section{Bibliographie :}

I1 A. BLAISE - C. LESUEUR - Sound transmission optimisation of some Ariane multilayered structures - $13^{\text {th }}$ International Congress on Acoustics - Belgrade Yougoslavie - 1989.

I2I A. BLAISE - C. LESUEUR - Acoustic transmission through 2D orthotropic multilayered infinite cylindrical shell - à paraître au J.S.V en Mars 1992.

I31 K.P. SOLDATOS - A refined laminated plate and shell theory applications - J.S.V 144(1) - 1991.

14I S. CHONAN - Sound proof characteristics of finite length orthotropic honeycomb shells - J.S.V $128(2)-1989$. 

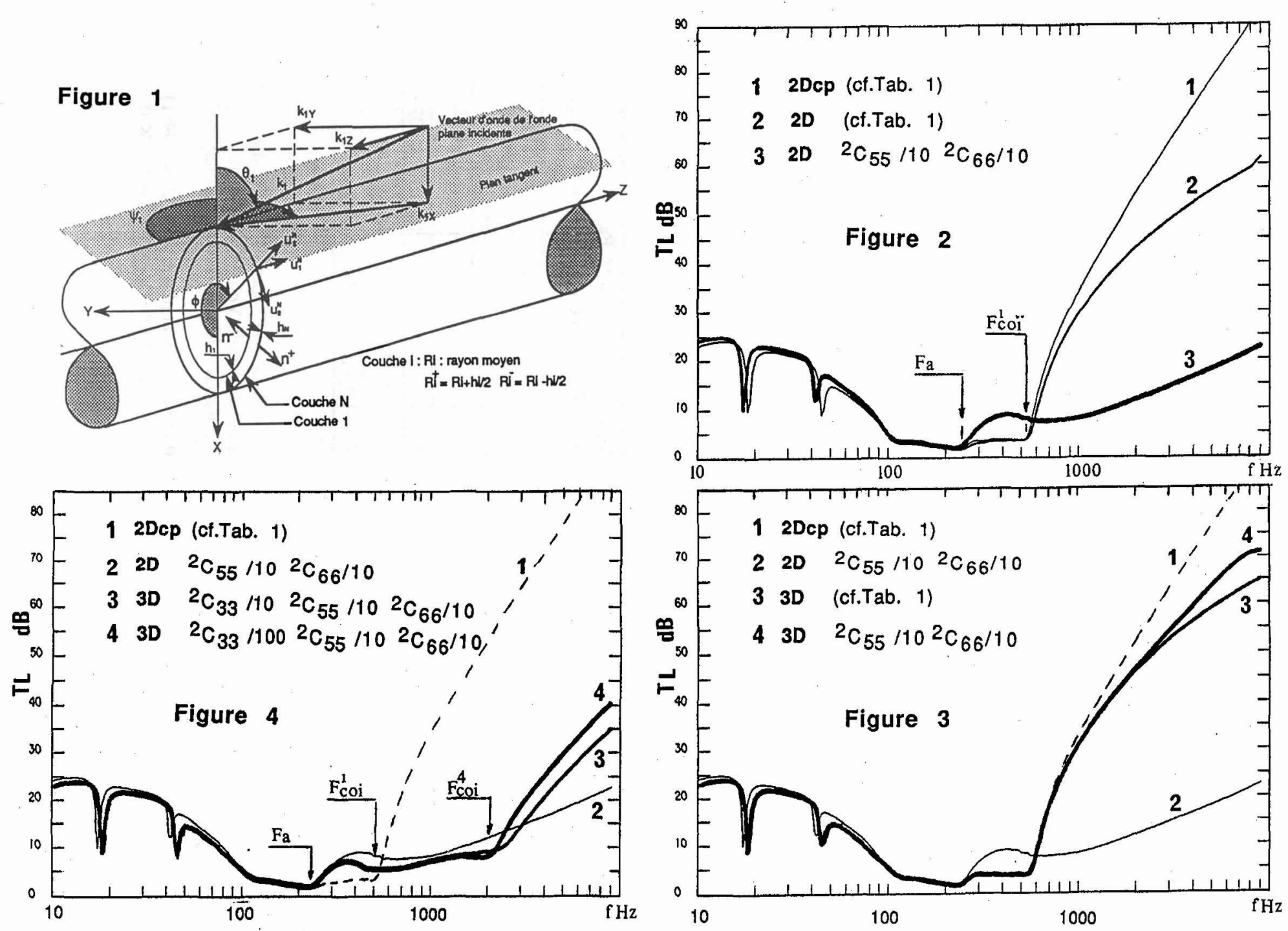

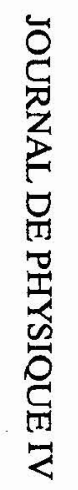

